\title{
DETERMINATION OF DENSITIES OF VALENCE STATES BY THE COMBINED USE OF UV AND X-RAY PHOTOEMISSION
}

\author{
N. J. SHEVCHIK, J. TEJEDA, M. CARDONA and D. W. LANGER \\ Max-Planck-Institut für Festkörperforschung, \\ Stuttgart, Bundesrepublik Deutschland
}

\begin{abstract}
Résumé. - La détermination de la densité d'états dans la bande de valence des solides par des études de photoémission est rendue plus efficace en utilisant à la fois de sources d'excitation dans l'ultraviolet lointain ( $h v=21,2$ et $40,8 \mathrm{eV})$ et dans le domaine des rayons X $(h v=1486,6 \mathrm{eV})$. L'utilisation de données provenant de ces deux sources d'excitation permet d'identifier et d'éliminer les effets de sections efficaces de photoionisation, de densité finale d'états, d'électrons secondaires et de résolution. On peut ainsi avoir une image plus correcte de la densité d'états que celle obtenue à partir de chacune de ces deux sources séparément. On montre aussi que l'utilisation de la raie à $21,2 \mathrm{eV}$ permet de déterminer de larges portions de la densité d'états de la bande de valence dans les composés II-VI avec plus de résolution qu'avec des rayons $\mathrm{X}$ monochromatiques.
\end{abstract}

Abstract. - The use of both far UV ( $h v=21.2$ and $40.8 \mathrm{eV})$ and X-ray ( $h v=1486.6 \mathrm{eV})$ excitation sources is shown to expand the power of photoemission experiments in determining the density of valence states in solids. When data from both excitation sources are used, the effects of photoionization cross-sections, final densities of states, secondary electrons, and resolution can be identified and eliminated. Thus, a better picture of the density of states can be obtained than with each source used independently. It is also shown that the $21.2 \mathrm{eV}$ line can be used to determine large portions of the density of valence states in II-VI compounds with better resolution than can be obtained with monochromatized X-rays.

High energy photoelectron spectroscopy is now a well established, valuable technique for determining the density of valence states in solids [I]. The most troublesome difficulties remaining in the extraction of the density of valence states from the photoemission data arise from instrumental resolution, energy dependent photoionization cross sections, structure in the final density of states, and contributions from inelastically scattered electrons. Most of the recent works on semiconductors have been performed on complex experimental systems using either synchrotron radiation [2] or monochromatized X-rays [3] as excitation sources. Less ideal, but simpler experimental systems, equipped with a helium discharge lamp or a non-monochromatized X-ray tube, have not been used with as much success in the past.

Here, we wish to show that by retaining the best features of data obtained from a system equipped with a differentially pumped helium discharge lamp and a standard Al X-ray tube, we can overcome many of the difficulties hindering the interpretation of the data. Especially, we wish to show that with these sources used together, we can greatly expand the power of photoemission experiments in determining the electronic structure of solids.

We have listed in table I some typical data of the photoelectron spectra provided by the helium dis- charge lamp (He I and $\mathrm{He}$ II) and the $\mathrm{Al} \mathrm{X-ray} \mathrm{tube}$ $(\mathrm{AlK} \alpha)$. Other gases, such as neon (Ne I, $17 \mathrm{eV}$; $\mathrm{Ne}$ II, $27 \mathrm{eV}$ ) can be used in the discharge lamp. Here we consider only helium since it provides the most commonly used and the most useful type of excitation. The dominant line in each spectrum occurs at energies of $21.2,40.8$ and $1486.6 \mathrm{eV}$, thus providing a wider range than has been used in experimental systems equipped with a synchrotron source [2]. With a wide range of energies available one can infer variations in the photo-ionization cross sections across the bands. Also, since lighter contaminating elements rapidly lose their photoelectric cross sections in the X-ray region, the X-ray spectra are less subjected to their effects. Agreement found with all three spectra ( $\mathrm{He} \mathrm{l,} \mathrm{He} \mathrm{II,} \mathrm{and} \mathrm{AlK} \alpha$ ) assures us that the density of valence states characteristic of the material is obtained. Each spectrum has a weaker component occurring at higher energies than the stronger component ; for the $\mathrm{He} \mathrm{II}$ and $\mathrm{AlK} \alpha$ spectra, the weaker component has $10 \%$ of the strength of the stronger component and for the He I spectra, it has $2 \%$ of the strength. Unfortunately, the electron energy distribution curves consist of the sum of electrons emitted by each component, thus some complications may arise in interpreting data obtained from them. As we shall see shortly, these weaker 
TABLE I

Data on the photoelectron spectra provided by the helium discharge lamp

( $\mathrm{He} \mathrm{I}$ and $\mathrm{He} \mathrm{II})$ and the aluminum X-ray tube $(\mathrm{A} 1 \mathrm{~K} \alpha)$

$\begin{array}{cccccc}\text { Spectrum } & \begin{array}{c}\text { Component } \\ (\mathrm{eV})\end{array} & \begin{array}{c}\text { Strength } \\ -\end{array} & \begin{array}{c}\text { Resolution } \\ (\mathrm{eV})\end{array} & \begin{array}{c}\text { Useful range } \\ (\mathrm{eV})\end{array} & \begin{array}{c}\text { Electron escape } \\ \text { depth }(\AA)\end{array} \\ \mathrm{HeI} & 21.2 & 1.0 & \overline{0.1} & 0-15 & 10 \\ \mathrm{He} \mathrm{II} & 23.5 & 0.02 & & & 5 \\ & 40.8 & 1.0 & 0.1 & 0-17 & 20 \\ \mathrm{AlK} \alpha & 48.4 & 0.1 & & & \\ & 1486.6 & 1.0 & 1.2 & 0-1480 & \\ & \left(\mathrm{~K} \alpha_{1,2}\right) & & & & \end{array}$

components become particularly troublesome for materials containing core levels lying within $10 \mathrm{eV}$ of the bottom of the valence band.

The narrow widths of the He I and He II exciting lines permit resolutions of $0.1 \mathrm{eV}$ to be obtained readily; with the $\mathrm{AlK} \alpha$ spectrum, the natural line width limits the resolution to a considerably worse value of $1.2 \mathrm{eV}$. Even with a monochromator, the resolution obtainable with the $\operatorname{AIK} \alpha$ spectrum is improved to only $0.5 \mathrm{eV}$ [3], still quite inferior to that obtainable with the UV sources.

The depths in energy to which these spectra can probe the occupied states are listed in the next to last column. The $21.2 \mathrm{eV}$ line probes to a depth of only $15 \mathrm{eV}$ because electrons with energies less than the work function, typically about $5 \mathrm{eV}$, cannot escape the sample. This $15 \mathrm{eV}$ range, however, is sufficient to observe large portions of the valence band for many materials. Also, sometimes the occurrence of a large tail of secondary electrons, the origin of which is not entirely clear, further reduces the energy to which useful data are obtainable. The range of the He II spectrum is limited for the study of valence bands to a depth of $\sim 17 \mathrm{eV}$ by the onset of emission from the He I spectrum. For the sharper and stronger core levels, their emission by the He II spectrum can be easily separated from the broader valence band emission of the He I spectrum so that the useful range is extended to about $35 \mathrm{eV}$. And, of course, the X-rays can probe the occupied states to depths of $1500 \mathrm{eV}$, enabling the positions of the core levels as well as the valence band to be obtained.

The escape depths of the valence electrons emitted by these excitation lines are very small : $20 \AA$ for the $X$-ray, $10 \AA$ for the He I spectra, and $5 \AA$ for the He II spectrum [1]. These short escape depths necessarily place severe requirements on the cleanliness of the surface of the sample and on the vacuum conditions. However, the frequent agreement found between theory and data obtained on clean samples with these lines, suggests that they yield bulk, rather than surface densities of states [2]-[8]. The smaller escape depth of electrons emitted by the $40.8 \mathrm{eV}$ line can be exploited to determine the influence of the surface on the emission spectra [8].

In this work we present a few selected examples from past work that illustrate some of the points just mentioned. In figure 1 , we present recent photoemission data from $\mathrm{ZnSe}$ taken with monochromatic [3] and non-monochromatic X-ray spectra and the He II spectrum. The data obtained with the monochromatized X-ray source have been shown to resemble the bulk density of states [3] and thus presently set

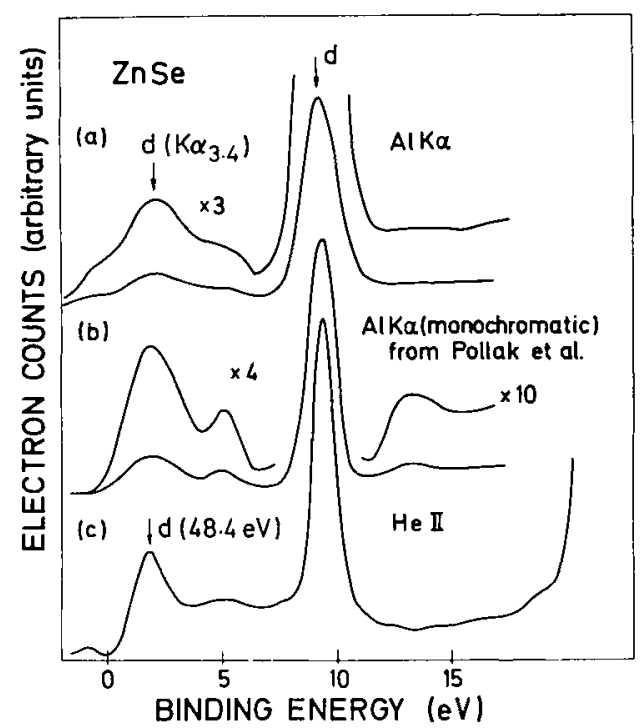

FIG. 1. - Photoemission data from sputtered $\mathrm{ZnSe}$ taken with monochromatic and non-monochromatic AlK $\alpha$ x-ray spectra and the He II spectrum. The monochromatic $X$-ray data are taken from [3].

the standard to be obtained here. The first peak arises from the upper p-like portions of the valence band; the large peak near $9.5 \mathrm{eV}$ from the $\mathrm{Zn} 3 \mathrm{~d}$ level, and the peak at $12 \mathrm{eV}$ from a band comprised of primarily the Se $4 s$ orbital. In the non-monochromatized X-ray data, the peak due to the $\mathrm{Zn} 3 \mathrm{~d}$ level is seen clearly but the top portions of the valence 
bands are not. The valence band structure emitted by the stronger $\mathrm{K} \alpha_{1,2}$ component is overlapped and obscured by the $3 \mathrm{~d}$ band structure emitted by the weaker $\mathrm{K} \alpha_{3,4}$ component, which is absent in the monochromatized X-ray source. It is doubtful whether the density of valence states can ever be extracted confidently from such data, even by a careful subtraction of the shape of the emission from $K \alpha_{3,4}$ component. The $\mathrm{He}$ II spectra similarly shows the large peak from the $3 \mathrm{~d}$ level, but as in the X-ray photoemission curve, the $\mathrm{d}$ band emitted by the weaker component of the He II spectra obscures the valence band emitted by the stronger component. Though the valence band density of states is difficult to obtain from these data, the $d$ bands are better resolved than in the monochromatized XPS data.

However, the data obtained with the He I spectrum, shown in figure 2 , are very similar to those obtained with the monochromatized X-rays, particularly after we subtract from the former the linearly rising background due to secondary electrons. The

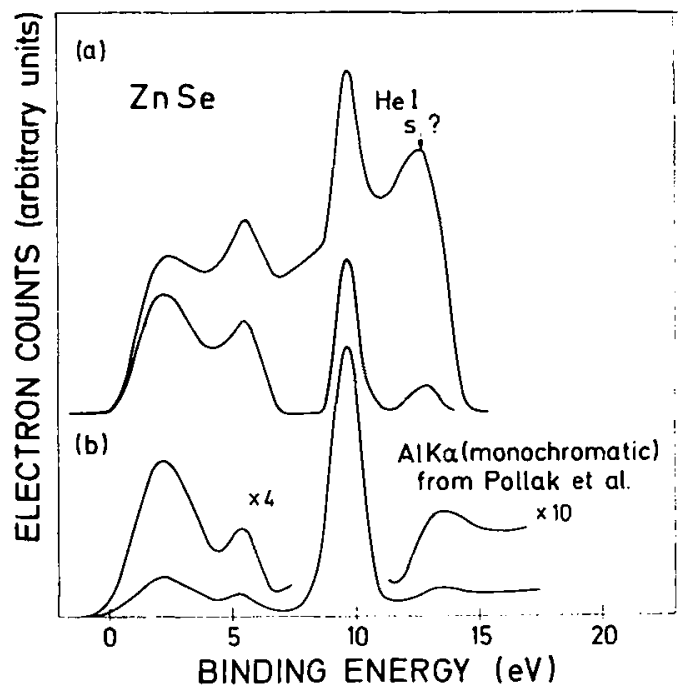

Fig. 2. - Comparison of the photoemission data taken with (a) the He I spectrum and (b) monochromatic $X$-rays, from [3]. The lower curve in (a) is found by subtracting a linearly rising background from the upper curve.

success of the $\mathrm{He}$ I line can be expected because of its better spectral purity and because of the larger photoionization cross section of the valence band relative to the $d$-bands for this excitation energy. The lower s bands, however, are not as well resolved. The small peak near the work function cutoff might be associated with this band, but it would be dangerous to make this assignment conclusively. The agreement between the UV and X-ray curves of figure 2 assures us that structure in the final density of states is unimportant, even for the low $21.2 \mathrm{eV}$ excitation energy. This similarity also shows that we can do as well in obtaining the density of valence states as those using the more complex monochromatized X-ray systems. More important, not only is the resolution superior, but the time required to collect the UV data shown in figure 2 is only about five minutes; in order to obtain the same statistics with an X-ray source at least a factor of 10 increase in the collection time is necessary.

Now let us consider trigonal Se (see Fig. 3), an example with no core levels in the immediate vicinity of the valence bands. When this is the case, we can anticipate all three excitation lines to give useful information. At first glance one sees that the top portions of the valence band are obtained with each excitation line. However, the $\mathrm{He}$ I spectrum only yields the top portion of the valence bands because

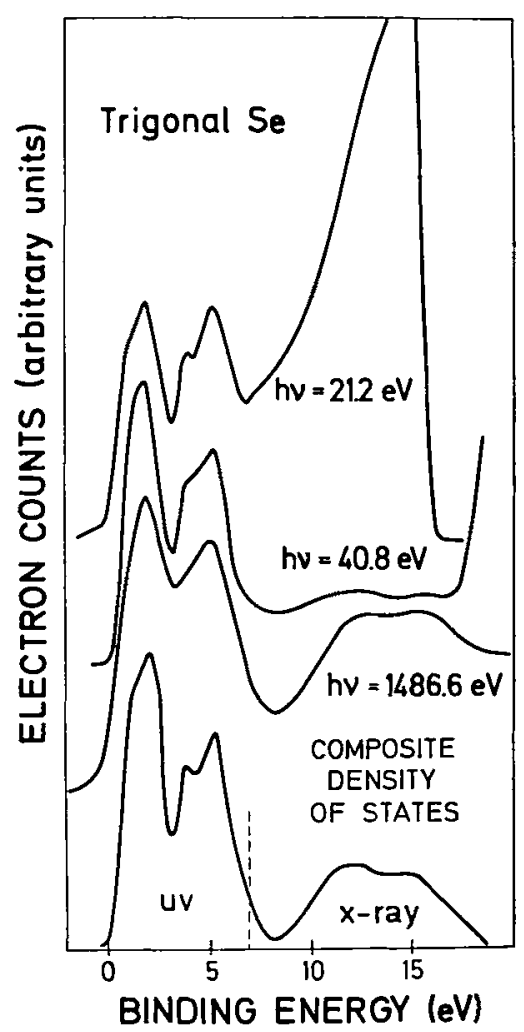

FIG. 3. - Photoemission data from trigonal selenium taken with the $\mathrm{He} \mathrm{I}, \mathrm{He}$ II and $\mathrm{AlK} \alpha$ spectra. The lowest curve is a composite density of valence states deduced from the 21.2 and $1486.6 \mathrm{eV}$ excitation lines. See [4] and [8].

of the rapidly rising secondary tail that obscures any structure at lower binding energies. The $s$ bands appear weakly in the He II data, but the onset of the emission from the He I spectrum does not permit us to see their lower portions. In the X-ray data, however, the $s$ bands are considerably stronger than in the above cases and we are able to determine their shapes easily. By combining the data obtained from the He $I$ and X-ray spectra, we deduce a composite density of states which retains the best features from each spectrum, especially the high resolution of the UV data. Thus, we are able to obtain the entire density of valence states by exploiting the wide range of excitation energies available. It is also encouraging 
to find agreement with the three excitation lines, confirming their independent abilities to expose the density of valence states.

The next example, amorphous $C$ prepared by sputtering, shown in figure 4 , demonstrates more dramatically the variations that can be found in the photoionization matrix elements across the valence band in the XPS data. The dotted line in this curve is taken to be the contribution from secondary electrons. We note at low binding energies that the X-ray photoemission is smaller than we would expect if the photoelectric matrix elements were energy independent. Thus, from the $\mathrm{X}$-ray data alone, it would be difficult to obtain the undistorted density of valence states. However, with the He II spectra, there seems

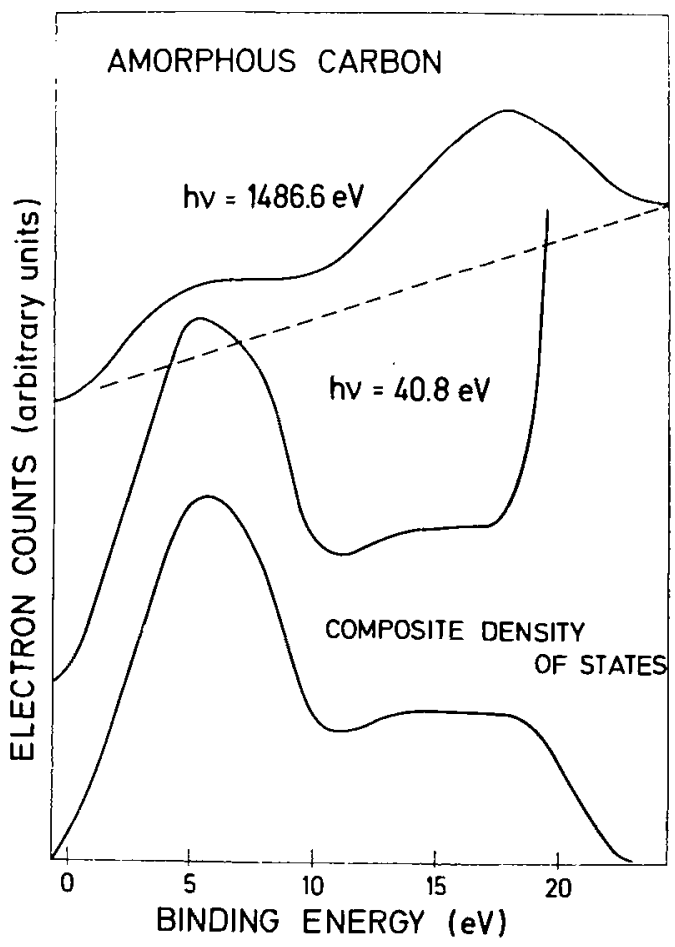

Fig. 4. - Photoemission data from amorphous carbon taken with the 1486.6 and $21.2 \mathrm{eV}$ excitation lines. The lower curve is a composite density of states deduced from the above data.

to be no strong variation in the matrix elements across the valence band. The lower portion of the valence band cannot be seen in the UV data because of the onset of the emission from the He I spectrum, but it is clearly seen in the XPS data. Combining the lowest $5 \mathrm{eV}$ of the X-ray data (a range in which the matrix elements are not so rapidly varying) with the UV data we obtain the composite density of states shown in figure 4 . In this case, the complete density of states could not have been obtained without the availability of both excitation sources.

With the addition of the X-ray source the scope of the research can be expanded beyond investigations of the density of valence states. The large energy of the X-ray excitation line permits the positions of the core levels to be determined, giving us information about chemical shifts between compounds and pure elements. These chemical shifts are related, in some way, to ionicity differences and thus they can, in principle, provide tests for the ionicity scales currently available [9]-[12]. With the simultaneous use of UV photoemission, the top of the valence band, determined to an accuracy of $0.1 \mathrm{eV}$, can be used as a reference point, eliminating uncertainties in the Fermi level and the effects of charging.

Since the escape depths of the electrons emitted by the three excitation lines are less than $30 \AA$, the cleanliness of the surface is important. Such cleanliness can be achieved by cleaving crystals in vacuum or by preparation in situ by evaporation or sputtering. The latter two methods are particularly subjected to contamination, and thus monitoring of the chemical composition of the deposited films becomes mandatory. A great advantage of having the $X$-ray source available is that most contaminating elements can be detected in the core level data.

An important bi-product of the X-ray data is the loss tail following the emission peaks from core levels. A typical example is shown in figure 5 for $\mathrm{Mg}$ in which 5 plasmon losses can be seen. These results are

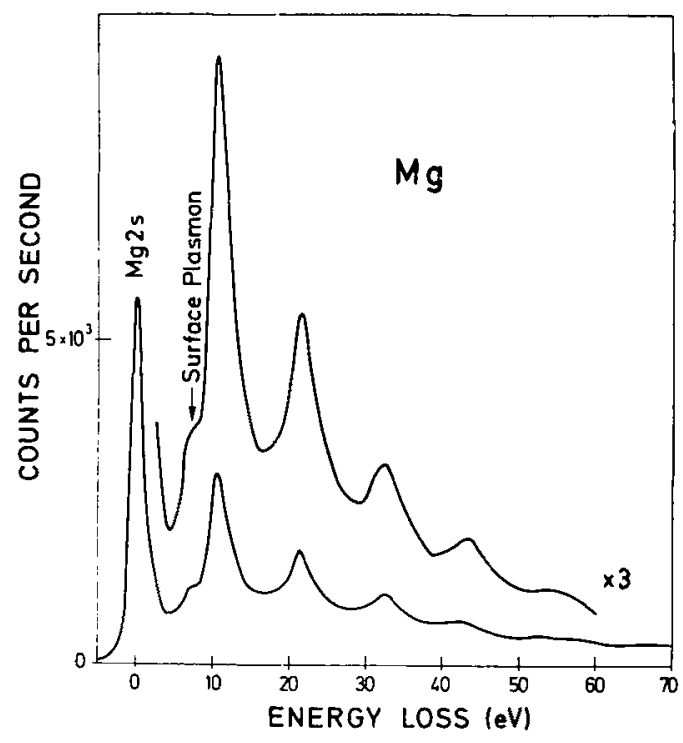

FIG. 5. - The energy loss spectrum following the emission from the $\mathrm{Mg}$ 2s level (from [16]).

very similar to those found by others using the backscattering technique with electron excitation [14]. Plasmon frequencies determined from these spectra have been found useful in detecting density differences between amorphous and crystalline materials [8], [13]. Also, they are of use in making secondary electron corrections to the valence bands determined by XPS and UPS [7].

Data obtained from another process, Auger recom- 


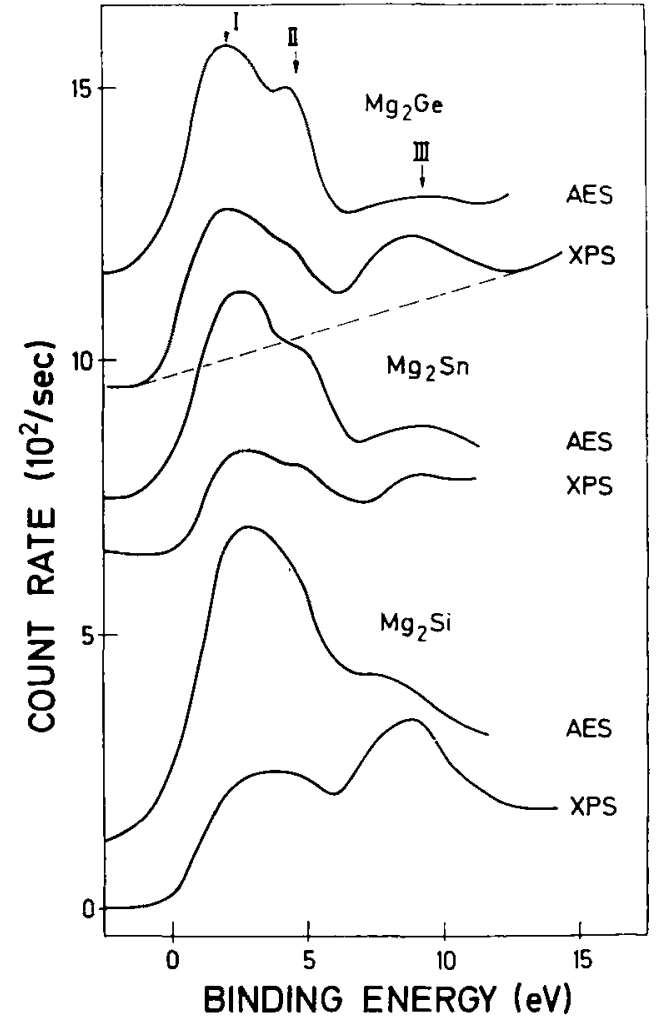

FIg. 6. - XPS and AES spectra of the valence bands of $\mathrm{Mg}_{2} \mathrm{Si}$, $\mathrm{Mg}_{2} \mathrm{Ge}$ and $\mathrm{Mg}_{2} \mathrm{Sn}$ (from [16]). bination, also yield information on the density of valence states [15], [16]. In figure 6, we compare the $X$-ray photoemission data from $\mathrm{Mg}_{2} \mathrm{Sn}, \mathrm{Mg}_{2} \mathrm{Ge}$, and $\mathrm{Mg}_{2} \mathrm{Si}$, and the Auger emission data from the $\mathrm{Mg}$ atom in these compounds [15]. In this Auger process, a hole in the $\mathrm{MgK}$ shell is created by the $\mathrm{AlK} \alpha \mathrm{X}$-ray photons. An electron in the higher lying $L$ shell falls into this hole while emitting an energy conserving electron from the valence band. Since the two core levels involved have well defined energies, the structure in the Auger spectrum primarily reflects structure in the density of valence states. The important advantage of the Auger process is that it samples the charge density about the emitting atom. The lower portions of the valence bands are weaker in the Auger data because the $\mathrm{Mg}$ atom participates less strongly in the bonding at lower binding energies.

Conclusion. - We hope to have shown that the combined use of UV and X-ray excitation sources expands the power of photoemission experiments in determining the electronic structure of solids. More importantly, we have shown that in some cases, a simple system equipped with a helium discharge lamp and an Al X-ray tube can do as well as those more complex systems employing monochromatized $\mathrm{X}$-rays and synchrotron radiation.

\section{References}

[1] LANGER, D. W., Festkörperprobleme XIII, Advances in Solid State Physics (Pergamon Press, Viewweg, 1973) in press.

[2] Eastman, D. E. and Grobman, W. D., Phys. Rev. Lett. 28 (1972) 1327 ; also see the paper presented by D. E. EASTMAN in this volume.

[3] Pollak, R. A., Ley, L., Kowalczyk, S., Shirley, D. A., Joannopoulos, J. D., Chadi, D. J. and Cohen, M. L., Phys. Rev. Lett. 29 (1972) 1103.

[4] Shevchik, N. J., Tejeda, J., Cardona, M. and Langer, D. W., Solid State Commun., 12 (1973) 1285.

[5] Kingston, D. L. and Vesely, C. J., Phys. Rev. B, in press.

[6] Cardona, M., Langer, D. W., Shevchik, N. J. and Tejeda, J., Phys. Stat. Sol., B 58 (1973) 127.

[7] Shevchik, N. J., Tejeda, J., Penchina, C. M. and CarDONA, M., Solid State Commun. 11 (1972) 1619.

[8] Shevchik, N. J., Cardona, M. and Tejeda, J., Phys. Rev. $B$, in press.
[9] Vesely, C. J., HengehOld, R. L. and Langer, D. W. Phys. Rev. 135 (1972) 2296.

[10] Cardona, M., Penchina, C. M., Shevchik, N. J. and TejedA, J., Solid State Commun. 11 (1972) 1655.

[11] Vesely, C. J. and Langer, D. W., Phys. Rev. B 4 (1971) 451 .

[12] Cardona, M., Penchina, C. M., Koch, E. E. and Yu, P. Y., Phys. Stat. Sol. (b) 53 (1972) 327.

[13] Shevchik, N. J., Tejeda, J., Langer, D. W. and Cardona, M., Phys. Rev. Lett. 30 (1972) 654, and Phys. Stat. Sol., B 57 (1973) 245.

[14] Powell, C. J. and Swan, J. B., Phys. Rev. 115 (1959) 869.

[15] Tejeda, J., Shevchik, N. J., Langer, D. W. and Cardona, M., Phys. Rev. Lett. 30 (1973) 370.

[16] Tejeda, J., Cardona, M., ShevchiK, N. J., Langer, D. W. and Schönherr, E., Phys. Stat. Sol., B 58 (I973) 189. 